\title{
Psychological Impact of Living with Scars Following Burn Injury
}

Nancy E. E. Van Loey

\section{Contents}

48.1 Background -430

48.2 Psychological Problems After a Burn Injury - 430

48.3 Psychological and Social Impact of Living with Scars - 431

48.3.1 Body-Esteem and Self-Esteem Concerns - 431

48.3.2 Social Self-Consciousness of Appearance -431

48.4 Factors Impacting Adjustment - 431

48.4.1 Burn Severity and Scarring - 431

48.4.2 Facial Involvement -432

48.4.3 Concealed Scars - 432

48.4.4 Gender - 432

48.4.5 Importance of Appearance - 432

48.5 Interference of Psychological Problems with the Perception of the Scar -432

48.6 Management -433

48.7 Conclusion -433

References -434 


\subsection{Background}

There is no getting around it: a severe burn injury is a major life event that can dramatically disrupt a person's life. The traumatic nature of the event, the long hospitalization and co-occurring high pain intensity, and the suddenly changed appearance can have a profound impact on those affected. Along with medical advances, more people with severe burns survive their injury and live with scars in a society where a high premium is placed on physical appearance and attractiveness. Scars resulting from burns can have a conspicuous look and may capture attention from the environment. When scars are hypertrophic, they are typically firm, raised, red, and have a rough surface. Hypertrophic scars can be difficult to conceal when they are located at visible body parts. Also, scars in less visible areas can pose a burden on someone's life in specific situations such as the swimming pool, or when the weather is warm and more revealing clothing is indicated at that time.

Living with scars may become even more complicated in the current society. Professionals and academics are concerned about the changing notions of normality related to appearance and the increasing demands of beauty. A recent paper [1] mentioned that an increasing amount of people in the UK consult the plastic surgeon or use cosmetic products to look as good as possible. Pictures on social media are manipulated to present persons in the best possible way. Body image and the perception of bodies are changing and result in increasing engagement in beauty practices that are justified by the new concept of normality. Unsurprisingly, living in a world that values beauty can be challenging for those with a visible difference. It can be particularly challenging for the individual himself or herself when there is a high personal standard placed on beauty. Also, society may be less acceptant toward those with a visible difference. Therefore, even minor scarring may increase the call for plastic surgery and cosmetic interventions. Underlying psychological problems may therefore be a point of attention.

This chapter has the general purpose to provide information about the psychological impact of a burn injury and psychological difficulties that may be encountered when living with scars. Because survival rates have increased, more people live with the physical and psychological consequences of burns. Consequently, appropriate psychosocial support and interventions that help burn survivors deal with scars, from a personal view and the view from the outside world, become increasingly important. Also, an understanding care environment including all professionals, that is, medical doctors, nurses, and physiotherapist, can improve outcomes. Learning objectives of this chapter include to know the prevalence rates of diverse psychiatric disorders that can develop in the aftermath of burn injury, to be aware of signals that may require an in-depth examination of underlying problems, to be aware that psychological problems can affect the perceptions of scar outcomes, to have some knowledge of risk factors, and, finally, to have knowledge regarding psychological treatment options to support people living with scars.

\subsection{Psychological Problems After a Burn Injury}

A burn event happens suddenly and can be life threatening. This may elicit a broad range of psychological reactions. Although it is documented that a substantial group of patients has pre-burn existing psychological problems sometimes being the cause of the injury, for example, self-harm during psychosis or suicide attempts, one may also develop severe and long-term psychological problems in response to the trauma or the adjustment process to a changed appearance. In the acute phase of burn injuries, there is a large focus on physical recovery. Along with wound healing and with the stabilization of the physical condition comes the realization that scars may be permanent and that appearance has changed. Facing this change may elicit feelings of grief and diminished body and self-esteem.

Severe psychological disorders may be encountered such as posttraumatic stress disorder (PTSD), depression, and anxiety. These disorders can become chronic when persons do not receive appropriate treatment. PTSD is included in the Diagnostic and Statistical Manual of Mental Disorders, 5th Edition (DSM-V) as a stress disorder and can develop in response to a traumatic event. PTSD comprises four symptom clusters: intrusions (intrusive thoughts and memories), avoidance of trauma-related stimuli, alterations in arousal and reactivity, and negative alterations in cognitions and mood. Initial higher levels of acute stress symptoms may be expected and can be regarded as normal reactions to a distressing event. For a substantial proportion of the patients, the high initial stress levels largely decrease within the weeks or months following the trauma. Severe symptoms of acute traumatic stress are reported in approximately $25 \%$ of patients admitted to a burn center. In a subgroup, however, they persist and become chronic. Prevalence rates of full PTSD after 1 year postburn are around $10 \%$ and another $15 \%$ may display severe symptoms that do not meet all criteria, that is, partial PTSD.

Depression is a disorder typically characterized by a negative mood and diminished interest or pleasure dur- 
ing a period of 14 days or longer. One to 4 years after the burn event, about $10 \%$ fulfilled criteria of major depression with a postburn onset. Generalized anxiety disorder (GAD) entails the presence of excessive anxiety and worry, is experienced as challenging to control, and is accompanied by physical or cognitive symptoms. GAD was also found to occur in about $10 \%$ of burn survivors after 1 year. This implicates that over time, the majority of people involved in a burn injury appear to adjust well and they will recover from initial high stress and depressive symptoms but a substantial subgroup will have long-term problems [2]. Clinical attention and screening is needed for PTSD, depression, and anxiety.

\subsection{Psychological and Social Impact of Living with Scars}

After a burn injury, when wounds are healing and scars start to develop, one has to adjust to this new situation in which appearance has changed. There are two perspectives that have a mutual influence: the individual perspective and the social perspective. The first refers to how people look at themselves and the latter perspective entails the social perspective.

\subsubsection{Body-Esteem and Self-Esteem Concerns}

People sustaining a burn injury can be faced with noticeable visible differences resulting from large wounds that lead to scarring. Besides an altered appearance caused by these scars, functional limitations can occur if they are located across joints. Both the visible and functional characteristics can diminish satisfaction with appearance and can cause negative self-perceptions and difficulties with social interactions that place people at risk to develop depression and (social) anxiety disorders. These psychological problems may be debilitating to daily functioning in key areas of living such as occupational functioning.

Important psychological concepts such as selfesteem and body-esteem can be damaged when a person is forced to live with visible differences [3, 4]. Self-esteem is a generic cognitive representation of the self. It is a multidimensional concept comprising the view of the person on the different abilities and characteristics. It influences how external information is processed, for example remarks or looks from other people. Bodyesteem refers to the evaluation of one's own body and can be viewed as a part of self-esteem [5]. After acquired disfigurement, there is a change in body-esteem that a person needs to adjust to.
It is reasonable to expect that persons with more severe scarring have greater difficulty integrating the scars in daily life than a person with minor scarring. But objective characteristics such as severity of the burn injury in terms of body area affected, the number of surgeries needed, or the number of affected body parts are only modestly related to psychological well-being, indicating that psychological factors may be more important. One such factor, importance of appearance, showed to moderate the relationship between severity characteristics and body-esteem. This indicates that for those who attach little value to their appearance, scars have a lower impact on their body-esteem. For people who highly value appearance, scars negatively affect their body-esteem [6]. These aspects will be addressed in more detail below.

\subsubsection{Social Self-Consciousness of Appearance}

Scars can elicit negative reactions from the environment. This is well described after burns. Feelings of stigmatization is one of the documented social problems of living with scars. This feeling may result from reactions from other people such as prejudices, discrimination, being ignored, intrusive behaviors such as staring, intrusive questions and remarks, or even bullying [7]. People who have to deal with these reactions may develop a feeling of self-conscious and may perceive stigmatization which may affect self-esteem in a negative way. This in turn can induce avoidant behavior or sometimes (symptoms of) social anxiety. However, the impact of these reactions may differ across individuals. Some persons are more prone to feel stigmatized because of a stronger attentional bias toward stimuli that elicit fear. Psychological therapies can help dealing with these reactions and build self-esteem to increase quality of life.

\subsection{Factors Impacting Adjustment}

\subsubsection{Burn Severity and Scarring}

In general, the objective severity of scarring showed to be a poor predictor of psychological adjustment. Both small and large defects can elicit body image concerns and diminish satisfaction with appearance. Therefore, it has been argued that a person's subjective perception is a better starting point to assess how persons adjust [4]. However, the body of knowledge that shows (in)direct associations between injury severity and psychological problems is growing. One in-hospital study documented that there were no differences across groups with minor and severe burns, but dissatisfaction with appearance increased over 
time in the group with more severe burns [6]. Another study found that persons with severe burns showed to ruminate more often - rumination is considered a cognitive strategy in which perseverative negative thinking and impaired disengagement from negative stimuli is presentwhich in turn predicted the level of depressive symptoms [8]. However, permanent scarring can act as a reminder to the burn event and has the potency to maintain psychological problems. Particularly when persons developed posttraumatic stress symptoms in response to the burn event, changes in appearances can trigger re-experiencing the traumatic event, which is an important symptom of posttraumatic stress disorder [9]. This indicates that more severe burns, possibly associated with more functional problems, can partly drive psychological adjustment. This does not exclude the fact that less extensive scarring can also elicit appearance-related concerns. Paying attention to the role of the scars in personal life is therefore warranted.

\subsubsection{Facial Involvement}

The face is a key figure in identity and it plays a key role in personal communication and expression of emotions. Sustaining a severe facial burn can be a devastating experience as it affects issues that are central to identity and social interactions. It is commonly expected that persons with facial disfigurement have more problems compared to persons with scars located at body parts that can be covered with clothes. Although there is evidence that living with facial burns may be more distressing than non-facial burns, the evidence is not that straightforward. One of the explanations put forward as to why people with facial burns may adjust relatively well is that they get used to negative reactions and are therefore better able to anticipate these unwanted reactions. Still, it is well established that persons with facial injury are presented with certain social challenges. Unwanted social reactions include staring, intrusive questions and remarks, and prejudices. A study comparing persons with facial burns to persons with the face not involved found higher problems in several domains of functioning such as: emotions, that is, they perceived more anger and sadness, and they had more social problems due to appearance issues [10]. Examples are available illustrating that facial differences is given meaning by others, for example, a woman who is pitied because she is seen as a victim of abuse [11]. Due to these responses, the risk to develop social anxiety and avoidance, low mood, and difficulties with relationship may increase and remains a concern across the lifespan. This is illustrated by a quote from a 60 -year-old man who was injured during childhood: "There is a part of me that hasn't quite made the hurdle to $100 \%$ adjustment. I'm comfortable at home and work, and among my circle of friends. Going out among strangers brings good and bad days" [12], p. 368.

\subsubsection{Concealed Scars}

Scars that can be covered with clothes trigger dilemmas such as when to show the scars or how to present yourself in certain social situations, how to deal with stares in the swimming pool and at the beach. This can lead to avoidance of these situations because of the reactions of others. Hidden scars can also affect intimacy and sexuality, topics still surrounded with taboo. Scars can impact the ability to enjoy intimacy because one may not feel attractive and avoid being touched.

\subsubsection{Gender}

It is suggested that women are more frequently concerned about their appearance and therefore find it harder to cope with visible differences. Little research provides direct support for this assumption but, in general, there is evidence that women (with facial burns) or with more severe burns have higher depression scores and are less satisfied with appearance [13]. These findings do not exclude the fact that also (young) men can be troubled by (facial) disfigurement and can question their physical attractiveness. There is, however, evidence supporting that women were more concerned about the change in appearance. Men predominantly reported an increased awareness of their whole body and not specifically their appearance. This may indicate there is, in general, a higher risk for women to develop appearance concerns compared to men because appearance is more valued by women.

\subsubsection{Importance of Appearance}

How persons view themselves is closely related to how they feel. The role of scars in psychological problems after burns is therefore not straightforward. A person's appraisal of his or her appearance constitutes an important factor of self-esteem. There is evidence for the importance that persons attach to appearance in the adjustment process after burns. Importance of appearance showed to predict distress of living with burn disfigurement [6].

\subsection{Interference of Psychological Problems with the Perception of the Scar}

There is ample evidence that scar evaluation by health care providers does not match that of the patient and is a poor predictor of satisfaction with the treatment by patients. This may be frustrating for both the patient 
and the health care provider. Understanding why these differences occur may diminish disappointment for both. Psychological problems such as depression can alter the way scars are perceived. As shown in a study [14], many patients with burns were well able to score scar characteristics within the same range as the professional. Persons scoring lower on self-esteem, however, rated their scars more troublesome, compared to the professional's view. This particularly concerned hypertrophy, possibly due to the fact that this characteristic is most conspicuous. This indicates that the psychological state influences how scars are viewed. Particularly the discrepancy between the patient's and professional's view may be a starting point to evaluate psychological factors.

Bradbury [11] underlines the importance of recognizing psychosocial issues in surgical decision-making. Having a realistic view on surgical outcomes in terms of what can be expected and psychosocial factors that may interfere with expectations and decisions is important. She proposed a three-staged approach in which individuals working with patients with visible differences all have some knowledge on influencing psychosocial factors, which forms the first stage of attention. If indicated, patients can be referred to, for example, a clinical nurse specialist trained in counseling skills. In the event therapy is needed, referral to a psychologist should be considered. It is emphasized that understanding psychological needs surrounding surgery is imperative to ensure that personal needs are met.

\subsection{Management}

Over the last decades, a number of interventions has been described that have the objective to help people dealing with challenges that result from living with a visible difference. Social skills training was among the first intervention programs aimed at expanding the arsenal of responses to a variety of reactions of people. Other therapies include cognitive behavioral therapy (CBT) in which dysfunctional cognitions, appraisals, beliefs, and assumptions are identified and changed in order to learn more useful ways of dealing with the visible difference [11]. CBT showed to have the strongest evidence of effectiveness and can support patients to come to terms with the visible difference, it can help in decision-making, and showed to be effective in overcoming social anxiety [3]. An online program called YPFaceIt, which includes elements of CBT and social skills training, has shown improvement in persons with a visible difference [15], although more research in larger groups is needed.

Besides psychological therapies, peer support groups have shown to be beneficial to well-being of burn survivors. Talking to a person who experienced a similar event and is faced with the same challenges increases empowerment. This was illustrated in the next quote: "When you meet someone who really knows what you're going through, you can discuss things in a different way" [16]. It is assumed that peer support groups can facilitate social sharing, which is a specific type of social support in which illness-related emotions are shared.

\subsection{Conclusion}

In sum, a burn injury can have devastating consequences in terms of psychosocial well-being. To predict who will adjust well after a burn injury comprises a complexity of factors that should be taken into account. It is an interplay of resilience and vulnerability factors; it involves a dynamic process over time. Some indications, however, may be points of attention for professionals in burn care. How persons cope with the consequences strongly depends on the significance they place on the value of their physical appearance. Objective factors such as gender, burn extent, and the extent to which the scars are visible all showed to have some relation but are poor direct predictors of dealing with the impact of scars. However, there are relationships that are worth considering. If there is a considerable deviation between the professional's and the patient's view on outcome, it is worth starting a dialogue with the aim to identify the causes. There is also a higher risk for women to struggle with visible differences as they, more frequently than men, value appearance and beauty; appearance may make up a larger part of their self-esteem and self-worth when compared to men. In men, functional problems may cause more problems.

The challenge for the health care provider is to be aware of signals that point to underlying psychological difficulties and judge to what extent another (surgical) intervention adds to the well-being of the patient. A staged approach of professionals with knowledge of psychosocial problems associated with visible differences should become more custom as it is now. It is only when the patient receives understanding, and whether it is clear for all parties what is going on in the patient, that compliance, decision-making, and outcome can be improved. Interventions focusing at psychological components may be indicated and can make a difference in the final outcome. 
Take-Home Messages

- Pay attention to the role of the scars in personal life.

- A changed appearance can have psychological and social consequences.

- Pay attention to signals that indicate underlying psychosocial problems.

- Be aware of underlying psychosocial issues in surgical decision-making.

- Consider a stepwise approach to recognize psychosocial issues.

\section{References}

1. Widdows H, MacCallum F. The demands of beauty: editors' introduction. Health Care Analysis. 2018;26:207-219. Epub 2018/07/11

2. Ter Smitten MH, de Graaf R, Van Loey NE. Prevalence and comorbidity of psychiatric disorders 1-4 years after burn. Burns. 2011;37(5):753-61. Epub 2011/02/22.

3. Rumsey N. Psychosocial adjustment to skin conditions resulting in visible difference (disfigurement): what do we know? Why don't we know more? How shall we move forward? Int J Women's Dermatol. 2018;4(1):2-7. Epub 2018/06/07.

4. Rumsey N, Harcourt D. Body image and disfigurement: issues and interventions. Body Image. 2004;1(1):83-97.

5. Lawrence JW, Fauerbach JA, Thombs BD. A test of the moderating role of importance of appearance in the relationship between perceived scar severity and body-esteem among adult burn survivors. Body Image. 2006;3(2):101-11.

6. Thombs BD, Notes LD, Lawrence JW, Magyar-Russell G, Bresnick MG, Fauerbach JA. From survival to socialization: a longitudinal study of body image in survivors of severe burn injury. J Psychosom Res. 2008;64(2):205-12.
7. Shepherd L, Tattersall H, Buchanan H. Looking in the mirror for the first time after facial burns: a retrospective mixed methods study. Burns. 2014;40(8):1624-34. Epub 2014/04/20.

8. Van Loey NE, Oggel A, Goemanne AS, Braem L, Vanbrabant L, Geenen R. Cognitive emotion regulation strategies and neuroticism in relation to depressive symptoms following burn injury: a longitudinal study with a 2-year follow-up. J Behav Med. 2014;37(5):839-48. Epub 2013/10/16.

9. Macleod R, Shepherd L, Thompson AR. Posttraumatic stress symptomatology and appearance distress following burn injury: an interpretative phenomenological analysis. Health Psychol. 2016;35(11):1197-204. Epub 2016/10/18.

10. Ryan CM, Lee A, Stoddard FJ Jr, Li NC, Schneider JC, Shapiro GD, et al. The effect of facial burns on long-term outcomes in young adults: a 5-year study. J Burn Care Res. 2018;39(4): 497-506. Epub 2018/06/15.

11. Bradbury E. Meeting the psychological needs of patients with facial disfigurement. Br J Oral Maxillofac Surg. 2012;50(3): 193-6. Epub 2011/03/29.

12. Jones BA, Buchanan H, Harcourt D. The experiences of older adults living with an appearance altering burn injury: an exploratory qualitative study. J Health Psychol. 2017;22(3):364-74. Epub 2015/09/02.

13. Al Ghriwati N, Sutter M, Pierce BS, Perrin PB, Wiechman SA, Schneider JC. Two-year gender differences in satisfaction with appearance after burn injury and prediction of five-year depression: a latent growth curve approach. Arch Phys Med Rehabil. 2017;98(11):2274-9. Epub 2017/05/10.

14. Hoogewerf CJ, van Baar ME, Middelkoop E, van Loey NE. Patient reported facial scar assessment: directions for the professional. Burns. 2014;40(2):347-53. Epub 2013/10/22.

15. Williamson H, Griffiths C, Harcourt D. Developing young person's face IT: online psychosocial support for adolescents struggling with conditions or injuries affecting their appearance. Health Psychol Open. 2015;2(2):2055102915619092. Epub 2017/01/11.

16. Oster C, Kildal M, Ekselius L. Return to work after burn injury: burn-injured individuals' perception of barriers and facilitators. J Burn Care Res. 2010;31(4):540-50. Epub 2010/07/10.

Open Access This chapter is licensed under the terms of the Creative Commons Attribution 4.0 International License (http://creativecommons. org/licenses/by/4.0/), which permits use, sharing, adaptation, distribution and reproduction in any medium or format, as long as you give appropriate credit to the original author(s) and the source, provide a link to the Creative Commons license and indicate if changes were made.

The images or other third party material in this chapter are included in the chapter's Creative Commons license, unless indicated otherwise in a credit line to the material. If material is not included in the chapter's Creative Commons license and your intended use is not permitted by statutory regulation or exceeds the permitted use, you will need to obtain permission directly from the copyright holder.

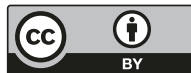

